\title{
Clinical Study \\ Predictive and Prognostic Factors in Definition of Risk Groups in Endometrial Carcinoma
}

\author{
Bengt Sorbe \\ Department of Oncology, Örebro University Hospital, 70185 Örebro, Sweden \\ Correspondence should be addressed to Bengt Sorbe, bengt.sorbe@orebroll.se \\ Received 28 August 2012; Accepted 27 September 2012 \\ Academic Editors: L. Gebrim and P. K. Mallmann
}

Copyright () 2012 Bengt Sorbe. This is an open access article distributed under the Creative Commons Attribution License, which permits unrestricted use, distribution, and reproduction in any medium, provided the original work is properly cited.

Background. The aim was to evaluate predictive and prognostic factors in a large consecutive series of endometrial carcinomas and to discuss pre- and postoperative risk groups based on these factors. Material and Methods. In a consecutive series of 4,543 endometrial carcinomas predictive and prognostic factors were analyzed with regard to recurrence rate and survival. The patients were treated with primary surgery and adjuvant radiotherapy. Two preoperative and three postoperative risk groups were defined. DNA ploidy was included in the definitions. Eight predictive or prognostic factors were used in multivariate analyses. Results. The overall recurrence rate of the complete series was $11.4 \%$. Median time to relapse was 19.7 months. In a multivariate logistic regression analysis, FIGO grade, myometrial infiltration, and DNA ploidy were independent and statistically predictive factors with regard to recurrence rate. The 5-year overall survival rate was 73\%. Tumor stage was the single most important factor with FIGO grade on the second place. DNA ploidy was also a significant prognostic factor. In the preoperative risk group definitions three factors were used: histology, FIGO grade, and DNA ploidy. Conclusions. DNA ploidy was an important and significant predictive and prognostic factor and should be used both in preoperative and postoperative risk group definitions.

\section{Introduction}

Endometrial carcinoma is the most common cancer of the female genital tract in the western world. Worldwide 287,000 new cases are diagnosed annually with this disease. Endometrial carcinomas are generally thought to have a favorable prognosis due to early detection, and the majority of tumors are detected in early stages. However, in fact this is not fully true, and there are important subgroups within this diagnosis with poor prognosis and outcome of treatment. Therefore, the first step to improve the situation has been to find predictive and prognostic factors, then to define clinically relevant risk groups, and finally to design clinical trials and treatment options for these risk groups.

Unfortunately, no consensus exists on which predictive or prognostic factors that should be used and how to combine them in the definition of suitable-risk groups. As a result of this, the randomized phase III trials presented during the last decades are difficult to compare since these definitions have varied, more or less, in most of them.
Another problem has been the small size and low power of most studies in the literature dealing with prognostic and predictive factors. Despite more or less sophisticated statistical methods with multivariate technique, the results are not reliable enough for definitive conclusions from such small series analyzing multiple variables. A few exceptions do exist but then with data from large registry studies, but then with other problems of selection and bias built in.

Six prospective randomized studies have been presented since 1980 to elucidate the value of external beam pelvic radiotherapy after surgery in early-stage endometrial carcinoma (Aalders, PORTEC-1, GOG\#99, ASTEC/EN.5, PORTEC-2, and Sorbe) [1-7]. The treated populations varied in all these studies from no risk groups defined (Aalders) [1] to a mixture of low-risk (PORTEC-1, GOG\#99) [2, 3], medium-risk (PORTEC-1, GOG\#99, ASTEC/EN.5, and PORTEC-2) [2-5], or high-risk cases (ASTEC/EN.5) [4]. Type of primary surgery and staging also varied from no staging at all (Aalders, PORTEC-1, ASTEC/EN.5, and PORTEC-2) $[1,2,4,5]$ to staging with lymph node sampling 
or complete lymphadenectomy (GOG\#99, ASTEC/EN.5) [3, 4]. Subgroup analyses performed within the frame of these studies have suffered from low power, and no level one data are presented for well-defined medium-risk or high-risk groups.

Three prospective randomized trials of low-risk, medium-risk, and high-risk cancers have been performed in Sweden and some other European countries. Vaginal brachytherapy, external beam pelvic radiation, and adjuvant chemotherapy were addressed in these studies. These three studies are now published [7-9].

In the present retrospective study a large, comprehensive, and consecutive series of more than 4,500 endometrial carcinomas in FIGO stages I-IV were analyzed with regard to predictive and prognostic factors and definition of the risk groups used in the above mentioned three prospective randomized studies. Special emphasis will be made on the prognostic value of DNA ploidy and the importance of this factor in the risk group definitions.

\section{Material and Methods}

2.1. Patients. One Swedish Cancer Center (Örebro) for gynecological oncology recruited patients with all stages (FIGO IIV) of endometrial carcinomas in an observation study. The period of recruitment was from January 1975 to December 2009. In all, 4,543 patients were included. Postoperative external pelvic irradiation and/or vaginal brachytherapy were administered to the majority of the patients. No further treatment-related details were analyzed in this study. The median age of the patients was 67 years (range 23-99 years). Tumor characteristics are presented in Tables 1 and 2.

2.2. Risk Group Definitions. The definition of high-risk carcinomas was as follows: (1) FIGO stage I, (2) nonendometrioid histological type, (3) presence of two of the following risk factors: FIGO grade 3 (poorly differentiated), deep $(\geq 50 \%)$ myometrial infiltration, DNA aneuploidy (FCM), (4) nuclear grade 3, (5) pathologically negative lymph nodes, and (6) negative abdominal cytology. Points 5-6 were optional in this study, and data are not available for all cases.

The definition of medium-risk carcinomas was as follows: (1) FIGO stage I, (2) endometrioid histological type, (3) presence of one of the following risk factors: FIGO grade 3 (poorly differentiated), deep $(\geq 50 \%)$ myometrial infiltration, DNA aneuploidy (FCM), (4) nuclear grade 12, (5) pathologically negative lymph nodes, and (6) negative abdominal cytology. Points 5-6 were optional in this study, and data are not available for all cases. Lymph vascular space invasion (LVSI) was not regularly included in the pathology reports at the participating centers and was not included in the definition of the medium-risk group.

The definition of low-risk carcinomas was as follows: (1) FIGO stage I, (2) endometrioid histological type, (3) presence of none of the following risk factors: FIGO grade 3 (poorly differentiated), deep $(\geq 50 \%)$ myometrial infiltration, DNA aneuploidy (FCM), or (4) nuclear grade 3. All pathology reports were reviewed by one experienced pathologist at the regional referral center.
TABle 1: Tumor stage (clinical and surgical) distributions of the complete series.

(a) FIGO stage (clinical)

\begin{tabular}{lc}
\hline IA & 1388 \\
IB & 865 \\
II & 185 \\
III & 108 \\
IV & 97 \\
None & 1900 \\
\hline Total & 4543 \\
\hline
\end{tabular}

1840 patients were staged both clinically and surgically.

(b) FIGO stage (surgical)

\begin{tabular}{lc}
\hline I* $^{*}$ & 899 \\
IA & 91 \\
IB & 1453 \\
IC & 625 \\
II* & 130 \\
IIA & 50 \\
IIB & 100 \\
III* & 82 \\
IIIA & 84 \\
IIIB & 11 \\
IIIC & 68 \\
IVA & 3 \\
IVB & 144 \\
None & 803 \\
\hline Total & 4543 \\
\hline${ }^{*}$ No further substage. & \\
&
\end{tabular}

2.3. Primary Surgery. The primary surgery was total abdominal hysterectomy, bilateral salpingo-oophorectomy, appendectomy, node sampling of enlarged lymph nodes, and peritoneal washing with cytology. Lymphadenectomy (pelvic \pm paraaortic) was not performed as a routine at the centers referring patients to the regional clinic. The surgery was performed at five departments of Gynecology and Obstetrics, but all patients were then referred to a Gynecologic Oncology Department for postoperative evaluation and treatment. The time interval between surgery and brachytherapy \pm external pelvic irradiation was 4-8 weeks. All patients were then planned for a 10-year follow-up program. The median follow-up period at the time of analysis was 115 months (range 1-362 months) for patients alive. During all visits, symptoms and signs related to the therapy were recorded, but in this study treatment-related side effects are not presented.

2.4. Brachytherapy. For the brachytherapy treatments, MicroSelectron HDR machines with an iridium source (Ir192) were used. Plastic vaginal cylinders with a diameter of $20 \mathrm{~mm}, 25 \mathrm{~mm}$, or $30 \mathrm{~mm}$ were used as standard. The diameter of the cylinder was individually chosen to ensure good contact between the surface of the applicator and the vaginal mucosa. The length of the vagina was measured from 
TABLE 2: Tumor characteristics of the complete series.

\begin{tabular}{|c|c|c|}
\hline \multicolumn{3}{|l|}{ Histology } \\
\hline Endometrioid & 3971 & $87.4 \%$ \\
\hline Nonendometrioid & 323 & $7.1 \%$ \\
\hline Unspecified & 249 & $5.5 \%$ \\
\hline \multicolumn{3}{|l|}{ Nuclear grade } \\
\hline 1 & 538 & $11.8 \%$ \\
\hline 2 & 746 & $16.4 \%$ \\
\hline 3 & 433 & $9.5 \%$ \\
\hline Unknown & 2866 & $62.2 \%$ \\
\hline \multicolumn{3}{|l|}{ P53 status } \\
\hline Positive & 249 & $5.5 \%$ \\
\hline Negative & 258 & $5.7 \%$ \\
\hline Unknown & 4036 & $88.8 \%$ \\
\hline \multicolumn{3}{|l|}{ Myometrial infiltration } \\
\hline Endometrium alone & 95 & $2.1 \%$ \\
\hline$\leq 50 \%$ & 1562 & $34.4 \%$ \\
\hline$>50 \%$ & 848 & $18.7 \%$ \\
\hline Unknown & 2038 & $44.9 \%$ \\
\hline \multicolumn{3}{|l|}{ FIGO grade } \\
\hline 1 & 1433 & $31.5 \%$ \\
\hline 2 & 1808 & $39.8 \%$ \\
\hline 3 & 850 & $18.7 \%$ \\
\hline Unknown & 452 & $9.9 \%$ \\
\hline \multicolumn{3}{|l|}{ DNA ploidy } \\
\hline Diploid & 1231 & $27.1 \%$ \\
\hline Nondiploid & 382 & $8.4 \%$ \\
\hline Unknown & 2930 & $64.5 \%$ \\
\hline
\end{tabular}

the vault to the level of introitus. The proximal $2 / 3$ of the vaginal length was defined as the target volume. The dose per fraction was specified at a depth of $5 \mathrm{~mm}$ from the surface of the vaginal cylinder with the HDR technique. Library dose plans that covered different vaginal lengths in steps of $10 \mathrm{~mm}$ and the different diameters of the cylinders were used. The dose calculations were made on the Nucletron Planning System (NPS v. 10) and the PLATO Brachytherapy Planning System (BPS v. 14) at centers using this equipment. Six fractions were given during an 8-day period. The dose per fraction was assigned to 2.5-3.0 Gy. Thus, the total doses delivered were 15.0-18.0 Gy. Recalculated to 2-Gy-equivalent doses $\left(E Q D_{2}\right)$, the total doses were 15.6-19.5 Gy at a depth of $5 \mathrm{~mm}(\alpha / \beta=10.0)$. All treatments were given on an outpatient basis.

2.5. External Beam Radiotherapy. External beam therapy was given to patients with high-risk tumors and to many with medium-risk tumors. The target volume was the previous site of the uterus and adnexa, the parametria, the proximal two-thirds of the vagina, and the lymphatic drainage regions along the iliac vessels up to the promontory. The superior field border was set at the L5-S1 disk. The total dose to be delivered to this volume was $46 \mathrm{~Gy}$ (median dose $46.0 \mathrm{~Gy}$, range 6-50 Gy) and daily fractions of 1.8-2.0 Gy (Table 3).
TABLE 3: Techniques used for the external beam pelvic radiotherapy.

\begin{tabular}{lc}
\hline Parameter & Specification \\
\hline Type of field & 4 -field box technique \\
Radiation quality & $6-50 \mathrm{MV}$ (linear accelerators) \\
$\begin{array}{l}\text { Dose per fraction } \\
\text { Number of fractions }\end{array}$ & $1.8-2.0 \mathrm{~Gy}$ \\
Total dose & $46.0 \mathrm{~Gy}$ (median) (range 5-26) \\
Fractionation & Daily fractions, 5 fractions per week \\
Superior field border & L5-S1 disk \\
Inferior field border & Lower margin of the fossa obturatoria \\
Lateral borders & $1 \mathrm{~cm}$ lateral of the linea terminals \\
\hline
\end{tabular}

2.6. Data Management. All data were collected in a computerized database at the Regional Oncology Center, Örebro, Sweden.

2.7. Statistical Analyses. In the statistical analyses, survival curves were generated using the Kaplan-Meier technique, and differences were tested with the log-rank test. The Pearson chi-square test was used for comparison of proportions and the independent $t$-test for comparing means of two groups. Multivariate analysis of prognostic factors was performed using the Cox proportional hazards model and logistic regression analysis. Best subset analysis was performed with multivariate technique to find the most important prognostic factors and to find the most powerful combination of these factors. All $P$ values were based on twosided tests, with $P<0.05$ considered statistically significant. The Statistica software package (StatSoft, Inc., Tulsa, OK, USA, version 10, 2010) was used for the statistical analyses.

\section{Results}

3.1. Recurrence Rate. The overall recurrence rate of the complete series was 519 out of 4,543 cases or $11.4 \%$. Eighty-seven vaginal recurrences (1.9\%) were diagnosed in the complete series. The regional pelvic (excluding vaginal recurrence) recurrence rate was $2.3 \%$ (103 cases), and the locoregional (vaginal or pelvic, or both) recurrence rate was 4.2\% (190 cases). Of 190 locoregional recurrences, 87 (46\%) occurred at the vaginal site. The 5-year actuarial locoregional relapse rate was 3.6\%. Distant recurrences (outside the pelvic area) were noted in 329 cases $(7.2 \%)$, and the 5 -year actuarial relapse rate was $6.6 \%$. The median time to relapse in the complete series was 19.7 months (range 1-248 months). In the complete series, 370 out of 519 recurrences $(71 \%)$ occurred within 3 years and 445 recurrences $(86 \%)$ within 5 years. The median age of all patients was 67.0 years (23-99 years), for those with recurrences was 68.4 years, and those without recurrences was 66.4 years.

3.2. Predictive Factors for Tumor Recurrences. At least 12 prognostic factors are described in endometrial carcinoma (Table 4). Some of them are also predictive factors for treatment outcome and tumor recurrences. Eight of these factors (age, FIGO stage, histology, FIGO grade, nuclear 
Table 4: Prognostic factors in endometrial carcinoma.

(1) FIGO stage (clinical, surgical)*

(2) Tumor size $(>2 \mathrm{~cm})$

(3) Histology (endometrioid, nonendometrioid)*

(4) Myometrial infiltration ( $>50 \%)^{*}$

(5) FIGO grade (grade 3 versus 1-2)*

(6) Nuclear grade (grades 1-3)*

(7) DNA ploidy (diploid, nondiploid)*

(8) S-phase fraction

(9) P53 expression (positive versus negative)*

(10) ER and PgR expression

(11) Lymphovascular space invasion (LVSI)

(12) Age of the patient (>60 years)*

*Factors analysed in the present study.

grade, DNA ploidy, myometrial infiltration, and p53 expression) were analyzed in this study with regard to the risk of tumor recurrence, both total rate and locoregional and distant recurrences. In a multivariate logistic regression analysis, three of these factors (FIGO grade, depth of myometrial infiltration, and DNA ploidy) were independent and statistically significant with regard to overall recurrence rate and distant recurrences (Table 5). The fourth most important predictive factor was the nuclear grade (best subset analysis). For locoregional recurrences no significant results were noted for these risk factors. In a model building analysis with best subset technique, the FIGO grade, depth of myometrial infiltration, and DNA ploidy gave the best predictive information with regard to the risk of tumor recurrences. Addition of further factors (age, histology, nuclear grade) only marginally increased the predictive value of the model. The single most important factor was the FIGO grade. Depth of myometrial infiltration was the second most important, and DNA ploidy (aneuploidy) the third factor. In this series $23.7 \%$ of the tumors with evaluable DNA status ( $n=1,613)$ were nondiploid (aneuploid).

3.3. Survival Analyses. At the last followup (March 2010), the number of patients alive was 2,764 (61\%), dead of disease $819(18 \%)$, and dead of intercurrent disease 960 (21\%). Death from intercurrent disease was more common than death from the cancer disease. The five-year actuarial overall survival rate was $73 \%$ and the cancer-specific survival rate was $83 \%$. Five-year overall survival after any relapse was $30 \%$. The salvage rate was $44 \%$ (38/87) after isolated vaginal recurrences, $20 \%(21 / 103)$ after pelvic recurrences, and $6 \%$ (19/329) after distant recurrences.

3.4. Prognostic Factors for Survival. Eight prognostic factors were analyzed with Cox proportional multivariate regression analyses and with overall and cancer-specific survival rate as the dependent variable. Seven of these factors were independent and statistically highly significant (Table 6). P53 expression, analyzed with immunohistochemistry, was the
TABLE 5: Multivariate logistic regression analyses of factors predicting recurrences.

\begin{tabular}{lccc}
\hline Factor & Odds ratio & $95 \%$ CI & $P$ value \\
\hline Overall tumor recurrences & & & \\
Age (>60 years) & 1.300 & $0.619-2.729$ & 0.489 \\
Histology* & 1.092 & $0.556-2.147$ & 0.798 \\
FIGO grade (3 versus 1-2) & 3.726 & $1.957-7.095$ & 0.00006 \\
Nuclear grade (3 versus 1-2) & 1.713 & $0.878-3.341$ & 0.114 \\
DNA ploidy** & 1.669 & $1.071-2.602$ & 0.024 \\
Myometrial infiltration*** & 2.077 & $1.392-3.098$ & 0.0003 \\
\hline Distant tumor recurrences & & & \\
Age (>60 years) & 0.844 & $0.377-1.889$ & 0.680 \\
Histology* & 1.155 & $0.572-2.330$ & 0.689 \\
FIGO grade (3 versus 1-2) & 4.750 & $2.319-9.729$ & 0.00002 \\
Nuclear grade (3 versus 1-2) & 1.686 & $0.809-3.515$ & 0.163 \\
DNA ploidy** & 1.805 & $1.101-2.957$ & 0.019 \\
Myometrial infiltration*** & 2.853 & $1.784-4.565$ & 0.00001 \\
\hline
\end{tabular}

${ }^{*}$ Nonendometrioid versus endometrioid. ${ }^{* *}$ Nondiploid versus diploid. $* * * 50 \%$ versus $<50 \%$.

only nonsignificant factor. Tumor stage (stages III-IV versus I-II) was the single most important factor with a risk ratio of 4.2 (95\% CI 3.5-5.0) for advanced tumor stage. Tumor grade (grade 3 versus 1-2) was the second most important prognostic factor with risk ratio 2.5 (95\% CI 2.1-3.0). Depth of myometrial infiltration had the lowest risk ratio $1.3(95 \%$ CI 1.1-1.6) among the seven significant risk factors. The nuclear grade of the tumor was significant and independent of the FIGO grade in multivariate analysis. DNA ploidy (aneuploid versus diploid) was also an important and significant prognostic factor with a risk ratio of 1.6 (95\% CI 1.3-2.0) with regard to cancer-specific survival rate.

3.5. Risk Group Definitions. The risk group definitions presented under Material and Methods were used in the complete series and for all stages together and for stage I alone. In the complete series, $54 \%$ of the cases fulfilled lowrisk criteria, 23\% medium-risk criteria, and 22\% high-risk criteria. In stage I, the corresponding figures were 57\%, 25\%, and $17 \%$, respectively. The discriminating power (chi-square $=471.8 ; P<0.000001$ ) with regard to cancer-specific survival rate was very high both for the complete series and for stage I tumors alone. The 5-year survival rate in the high-risk group was only $50 \%$ in the complete series, and this group was very distinctly separated from the low-risk and mediumrisk groups (Figure 1). On the other hand, the difference in survival between the last two groups was only $10 \%$ at 5 years.

If instead only two risk groups are used, which are proposed for preoperative risk group definitions, a 30\% difference was noted in cancer-specific survival at 5 years, which was also highly statistically significant $(Z=22.948$; $P<0.000001)$. In FIGO stage I, the difference between the two groups was $20 \%$ (75\% versus $95 \%)(Z=12.980$; $P<0.000001$ ) (Figure 2). In the preoperative definitions, only three prognostic factors were used: histology (nonendometrioid versus endometrioid), FIGO grade (grade 3 
TABle 6: Multivariate Cox proportional regression analyses of prognostic factors.

\begin{tabular}{lcccc}
\hline Factor & Risk ratio & $95 \%$ CI & $P$ value \\
\hline Overall survival rate & & & \\
Age ( $>$ 60 years) & 2.951 & $2.467-3.531$ & $<0.00001$ \\
FIGO stage (III-IV versus I-II) & 2.489 & $2.169-2.855$ & $<0.00001$ \\
Histology* & 1.569 & $1.340-1.836$ & $<0.00001$ \\
FIGO grade (3 versus 1-2) & 1.814 & $1.590-2.069$ & $<0.00001$ \\
Nuclear grade (3 versus 1-2) & 1.558 & $1.286-1.888$ & $<0.00001$ \\
DNA ploidy** & 1.456 & $1.215-1.744$ & $<0.0001$ \\
Myometrial infiltration*** & 1.322 & $1.164-1.501$ & $<0.0001$ \\
P53 expression**** & 0.955 & $0.715-1.276$ & 0.755 \\
\hline Cancer-specific survival rate & & & \\
Age (>60 years) & 1.925 & $1.509-2.456$ & $<0.00001$ \\
FIGO stage (III-IV versus I-II) & 4.205 & $3.542-4.993<0.00001$ \\
Histology* & 1.717 & $1.410-2.090<0.00001$ \\
FIGO grade (3 versus 1-2) & 2.524 & $2.099-3.035<0.00001$ \\
Nuclear grade (3 versus 1-2) & 1.635 & $1.279-2.090$ & $<0.0001$ \\
DNA ploidy** & 1.591 & $1.267-1.999$ & $<0.0001$ \\
Myometrial infiltration*** & 1.292 & $1.069-1.561$ & 0.008 \\
P53 expression**** & 1.089 & $0.747-1.587$ & 0.657 \\
\hline
\end{tabular}

${ }^{*}$ Nonendometrioid versus endometrioid. ${ }^{* *}$ Nondiploid versus diploid. $* * * 50 \%$ versus $<50 \%$. $* * * *$ Positive $(>30 \%$ staining) versus negative ( $<30 \%$ staining).

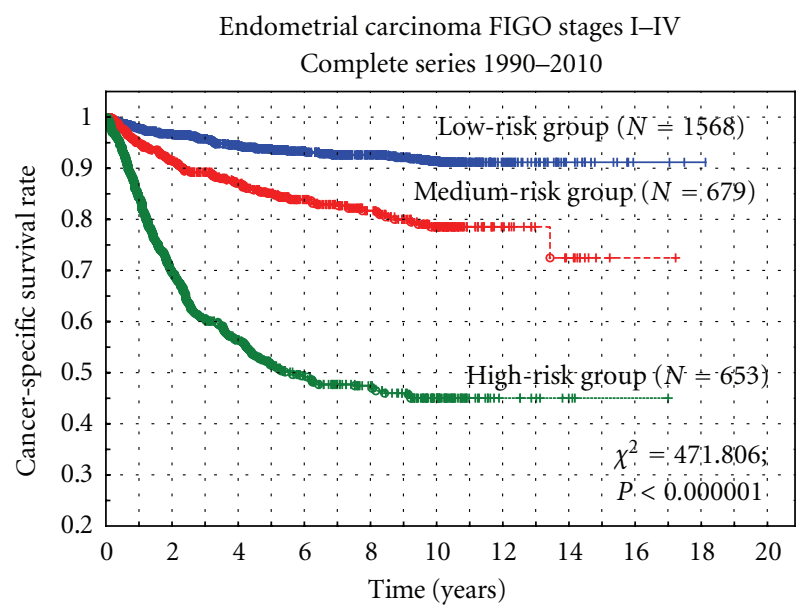

Figure 1: Cancer-specific survival rate versus three postoperative risk groups.

versus 1-2), and DNA ploidy (nondiploid versus diploid). It was not necessary to use myometrial infiltration, which is not a reliable prognostic factor assessed preoperatively.

\section{Discussion}

The optimal treatment of endometrial carcinoma patients have been vividly discussed and also studied in a number of randomized trials during the last decades [1-9]. Before that, no consensus existed with regard to type of therapy, but the situation has changed, and our evidence-based

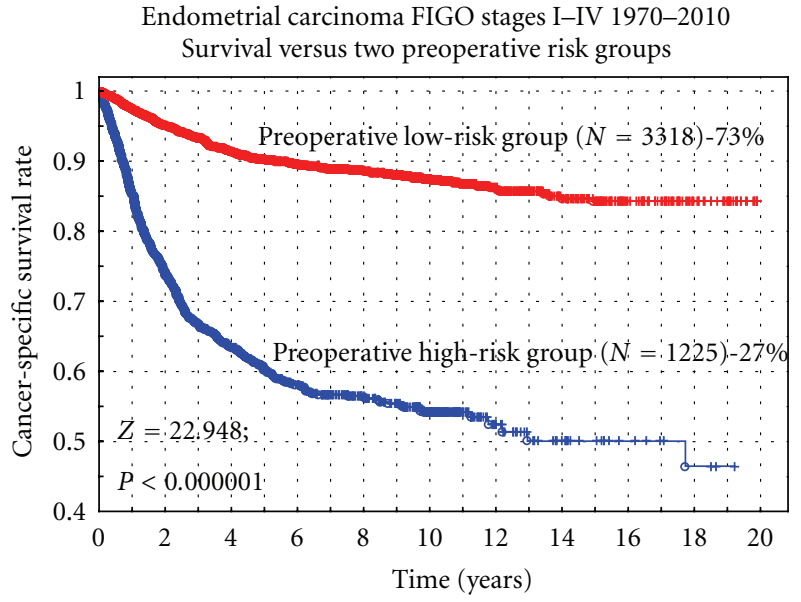

Figure 2: Cancer-specific survival rate versus two preoperative risk groups.

knowledge in this field has improved substantially. However, still different conclusions are drawn from the available study data, and the optimal treatment of the various risk groups is continuously debated in various countries and in different centers. Various definitions of the risk groups have confused the results, and conclusions drawn from the studies [25, 7-9]. The PORTEC-1 study [2] included both low-risk (grade 2, superficial infiltration) and medium-risk cases, and the ASTEC/EN.5 study [4] included both medium-risk and high-risk cases. Most authors agree that low-risk cases can be left with surgery alone $[7,10,11]$. Still, vaginal brachytherapy is effective and will reduce the rate of vaginal recurrences in all risk groups, but from different levels [7]. From a cost-effectiveness perspective, it seems reasonable to exclude the low-risk tumors from this type of adjuvant therapy. Treatment of isolated vaginal relapse after surgery alone is effective in $89 \%$ (complete remission) with $65 \%$ survival rate [12]. In the present study, the salvage rate was only $44 \%$ after vaginal recurrences. For medium-risk cases, the situation is not clear. A number of studies have focused on this risk group, but still with various definitions of this risk group (medium-risk, low-medium risk, and high-medium risk) [1-9]. A mixture of both low-risk and medium-risk cases has been studied as well as a mixture of medium-risk and high-risk cases. Improved locoregional tumor control has been shown but so far no influence on survival [1-8]. From our country a randomized study has presented data for a pure medium-risk group that did include neither lowrisk cases nor high-risk cases [9]. The low-risk and high-risk groups have been studied separately in two other randomized protocols, and the results have been presented elsewhere $[7,8]$.

The aim of the present study was to evaluate the prognostic value of the various clinical and histopathological factors commonly discussed in endometrial cancer and how to combine them into risk group definitions. Proposals of pre- and postoperative risk groups are presented and tested in a large series of endometrial carcinomas comprising more than 4,500 patients. The only large study published before 
analyzing prognostic factors in endometrial cancer was the registry SEER study, where 41,120 cases were included. FIGO stage, type of histology, FIGO grade, lymph node status, age at diagnosis, and race were found to be prognostic factors in that study [13].

The 5-year actuarial locoregional recurrence rate was $3.6 \%$, and the distant recurrence rate was $6.6 \%$ in our series. Eight of twelve commonly used prognostic and predictive factors were analyzed in this study. With regard to recurrences FIGO grade, DNA ploidy, and depth of myometrial infiltration were independent and significant predictive factors in a multivariate logistic regression analysis. A best subset analysis also confirmed that these three factors were the most important ones, and addition of further factors only marginally improved the predictive value of the model. The single most important factor was the FIGO grade, but it is important to point out that the DNA ploidy, not so commonly used in the international literature, was one of the three most important predictive factors together with myometrial invasion to predict the risk of tumor recurrences, and especially distant recurrences. A number of studies from Sweden [14-17] have pointed out the prognostic importance of DNA ploidy before, but this information does not seem to have been generally accepted and spread worldwide [2-5].

The 5-year actuarial overall survival rate in this series of patients was $73 \%$, and the cancer-specific survival rate was $83 \%$. The study covers a long time period, but in fact the overall survival did not change during the last three decades. Changes in the treatment technique during these years seem to have had no impact on survival. Cox proportional multivariate regression analysis was used to find out the most important prognostic factors with regard to the cancerspecific survival probability. Eight factors were included in the model, and seven were found to be independent and significant. Of the included factors only p53 expression was nonsignificant in these analyses. Advanced versus early tumor stage was the single most important factor with a risk ratio of 4.2, and FIGO grade was the second most important with a risk ratio of 2.5 . Interesting findings were that the nuclear grade $[14,15]$ was significant and independent of the FIGO grade, and the DNA ploidy with a risk ratio of 1.6 was more important than myometrial invasion with a risk ratio of 1.3. In fact, myometrial invasion had the lowest risk ratio of all seven analyzed and significant prognostic factors.

Tumor size and lymphovascular space invasion (LVSI) were not included in the present analyses, since these variables were not regularly reported by the departments of pathology during the extensive study period. Tumor size with a cutoff level of $2 \mathrm{~cm}$ has been reported to be an important predictive factor in preoperative risk classification to define a low-risk group where lymph node dissection is not required [18]. In another study tumor size was not an independent predictor of recurrence [19]. In a number of studies, LVSI has been pointed out as an important predictive factor associated with lymph node metastases and distant tumor spread [2022].

Three risk groups were analyzed with the definitions used in our country during the last 20 years and also in three published randomized multicenter studies [7-9].
Interesting to note is that $22 \%$ of all tumors belonged to a high-risk group with these definitions and 54\% belonged to a low-risk group. The prognoses of the three groups are highly significantly different, and especially the highrisk group showed a poor prognosis with only $50 \%$ cancerspecific survival rate. These definitions and risk groups seemed to work out well to discriminate between patients, where surgery alone is enough (low-risk cases), where vaginal brachytherapy should be added (medium-risk cases), and where external beam radiotherapy and chemotherapy probably are the treatment options [7-9].

For preoperative risk group classification it is more convenient to use two risk groups. The aim of this classification is to sort out those patients requiring lymph node dissection from those who do not. Myometrial invasion is an important predictive and prognostic factor but difficult to assess preoperatively in a reliable way. Our multivariate analyses of this large series of patients have shown that myometrial invasion can be replaced by other prognostic factors without losing to much of prognostic information. The results from our analysis showed that histology (nonendometrioid versus endometrioid), FIGO grade (grade 3 versus grade 1-2), and DNA ploidy (nondiploid versus diploid) could be used to define two preoperative risk groups. These two risk groups discriminated well $(P<0.000001)$ between low-risk and high-risk cases with a $30 \%$ difference in 5year cancer-specific survival rate. Using this definition, the preoperative high-risk group includes $27 \%$ of all new cases of endometrial cancer. In an Italian study, preoperative risk classification was made using histology, tumor grade, myometrial invasion, cervical spread, and abdominal spread and correctly identified the postoperative risk classification in $96 \%$ with high sensitivity and specificity [23]. This may help the surgeon in the decision to perform limited or extended surgery.

The importance of DNA ploidy as a predictive and prognostic factor in endometrial carcinoma $[14-17,24]$ and part of risk group classifications [7-9] is one of the most important results of this study. It is important to analyze large samples of endometrial carcinomas to sort out the most important and significant predictive and prognostic factors that should be used in future risk group classifications. It is also important for coming randomized studies that there will be an international consensus regarding the definition criteria to be used for the various risk groups.

\section{Conclusions}

Risk group definitions are important in the design of randomized studies in endometrial carcinomas. Up to now some confusion exists in these definitions in published randomized studies making firm conclusions and comparisons difficult. Three risk groups seem reasonable to use in the postoperative setting, but probably only two in the preoperative classification. Our study has shown that DNA ploidy is an important predictive and prognostic factor and if used in combination with the FIGO grade and type of histopathology can replace myometrial invasion in definition of preoperative high-risk cases needing more extensive surgery. 


\section{Conflict of Interests}

The author declares that there is no conflict of interests.

\section{Acknowledgment}

The author forwards great thanks to Peter Jansson, IT Coordinator at the Department of Oncology, Örebro University Hospital, for his work with the data base and retrieval of the patient data included in this study.

\section{References}

[1] J. Aalders, V. Abeler, P. Kolstad, and M. Onsrud, "Postoperative external irradiation and prognostic parameters in stage I endometrial carcinoma. Clinical and histopathologic study of 540 patients," Obstetrics and Gynecology, vol. 56, no. 4, pp. 419-427, 1980.

[2] C. L. Creutzberg, W. L. J. Van Putten, P. C. M. Koper et al., "Surgery and postoperative radiotherapy versus surgery alone for patients with stage- 1 endometrial carcinoma: multicentre randomised trial," The Lancet, vol. 355, no. 9213, pp. 1404$1411,2000$.

[3] H. M. Keys, J. A. Roberts, V. L. Brunetto et al., "A phase III trial of surgery with or without adjunctive external pelvic radiation therapy in intermediate risk endometrial adenocarcinoma: a Gynecologic Oncology Group study," Gynecologic Oncology, vol. 92, no. 3, pp. 744-751, 2004.

[4] P. Blake, A. M. Swart, J. Orton et al., "Adjuvant external beam radiotherapy in the treatment of endometrial cancer (MRC ASTEC and NCIC CTG EN. 5 randomised trials): pooled trial results, systematic review, and metaanalysis," The Lancet, vol. 373, pp. 137-146, 2009.

[5] R. A. Nout, V. T. H. B. M. Smit, H. Putter et al., "Vaginal brachytherapy versus pelvic external beam radiotherapy for patients with endometrial cancer of high-intermediate risk (PORTEC-2): an open-label, non-inferiority, randomised trial," The Lancet, vol. 375, no. 9717, pp. 816-823, 2010.

[6] R. A. Nout, H. Putter, I. M. Jürgenliemk-Schulz et al., "Quality of life after pelvic radiotherapy or vaginal brachytherapy for endometrial cancer: first results of the randomized PORTEC2 trial," Journal of Clinical Oncology, vol. 27, no. 21, pp. 35473556, 2009.

[7] T. Högberg, M. Signorelli, C. F. de Oliveira et al., "Sequential adjuvant chemotherapy and radiotherapy in endometrial cancer-results from two randomized studies," European Journal of Cancer, vol. 36, no. 2, pp. 371-378, 2010.

[8] B. Sorbe, B. Nordström, J. Mäenpää et al., "Intravaginal brachytherapy in FIGO stage I low-risk endometrial cancer: a controlled randomized study," International Journal of Gynecological Cancer, vol. 19, no. 5, pp. 873-878, 2009.

[9] B. Sorbe, G. Horvath, H. Andersson, K. Boman, C. Lundgren, and B. Pettersson, "External pelvic and vaginal irradiation versus vaginal irradiation alone as postoperative therapy in medium-risk endometrial carcinoma-a prospective randomized study," International Journal of Radiation Oncology, Biology, Physics, vol. 82, no. 3, pp. 1249-1255, 2012.

[10] H. K. Poulsen, M. Jacobsen, K. Bertelsen et al., "Adjuvant radiation therapy is not necessary in the management of endometrial carcinoma stage I, low-risk cases," International Journal of Gynecological Cancer, vol. 6, no. 1, pp. 38-43, 1996.

[11] A. Obermair, R. Cheuk, S. C. Pak et al., "Disease-free survival after vaginal vault brachytherapy versus observation for patients with node-negative intermediate-risk endometrial adenocarcinoma," Gynecologic Oncology, vol. 110, no. 3, pp. 280-285, 2008.

[12] C. L. Creutzberg, W. L. van Putten, P. C. Koper et al., "Survival after relapse in patients with endometrial cancer: results from a randomized trial," Gynecologic Oncology, vol. 89, pp. 201209, 2003.

[13] C. L. Kosary, "FIGO stage, histology, histologic grade, age and race as prognostic factors in determining survival for cancers of the female gynecological system: an analysis of 1973-87 SEER cases of cancers of the endometrium, cervix, ovary, vulva, and vagina," Seminars in Surgical Oncology, vol. 10, no. 1, pp. 31-46, 1994.

[14] B. Nordström, P. Strang, A. Lindgren, R. Bergström, and B. Tribukait, "Carcinoma of the endometrium: do the nuclear grade and DNA ploidy provide more prognostic information than do the FIGO and WHO classifications?" International Journal of Gynecological Pathology, vol. 15, no. 3, pp. 191-201, 1996.

[15] B. Sorbe and B. Risberg, "Prognostic importance of the nuclear proteins p53 and $\mathrm{Rb}$ in conjunction with DNA, nuclear morphometry and grading in endometrial carcinoma," International Journal of Gynecological Cancer, vol. 7, no. 1, pp. 34-41, 1997.

[16] A. Koul, P. O. Bendahl, A. Borg et al., "TP53 protein expression analysis by luminometric immunoassay in comparison with gene mutation status and prognostic factors in early stage endometrial cancer," International Journal of Gynecological Cancer, vol. 12, no. 4, pp. 362-371, 2002.

[17] C. Lundgren, G. Auer, B. Frankendal, B. Nilsson, and B. Nordström, "Prognostic factors in surgical stage I endometrial carcinoma," Acta Oncologica, vol. 43, no. 1, pp. 49-56, 2004.

[18] A. Mariani, M. J. Webb, G. L. Keeney, M. G. Haddock, G. Calori, and K. C. Podratz, "Low-risk corpus cancer: is lymphadenectomy or radiotherapy necessary?" American Journal of Obstetrics and Gynecology, vol. 182, no. 6, pp. 15061519, 2000.

[19] C. Shah, E. B. Johnson, E. Everett et al., "Does size matter? Tumor size and morphology as predictors of nodal status and recurrence in endometrial cancer," Gynecologic Oncology, vol. 99, no. 3, pp. 564-570, 2005.

[20] S. Vance, R. Yechieli, C. Cogan, R. Hanna, A. Munkarah, and M. A. Elshaikh, "The prognostic significance of age in surgically staged patients with Type II endometrial carcinoma," Gynecologic Oncology, vol. 26, no. 1, pp. 16-19, 2012.

[21] K. Narayan, P. Khaw, D. Bernshaw, L. Mileshkin, and S. Kondalsamy-Chennakesavan, "Prognostic significance of Lymphovascular space invasion and nodal involvement in intermediate- and high-risk endometrial cancer patients treated with curative intent using surgery and adjuvant radiotherapy," International Journal of Gynecological Cancer, vol. 22, pp. 260-266, 2012.

[22] A. Gadducci, A. Cavazzana, S. Cosio et al., "Lymph-vascular space involvement and outer one-third myometrial invasion are strong predictors of distant haematogeneous failures in patients with stage I-II endometrioid-type endometrial cancer," Anticancer Research, vol. 29, no. 5, pp. 1715-1720, 2009.

[23] F. Maneschi, I. Ceccacci, A. Perugini, C. Pane, A. Simeone, and A. Manicone, "Endometrial cancer: prognostic significance of risk classification based on pre-intraoperative findings," Archives of Gynecology and Obstetrics, vol. 285, no. 2, pp. 521527, 2011. 
[24] T. Song, J. W. Lee, H. J. Kim et al., "Prognostic significance of DNA ploidy in stage I endometrial cancer," Gynecologic Oncology, vol. 122, no. 1, pp. 79-82, 2011. 


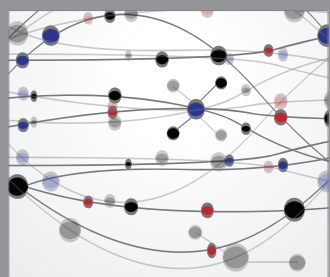

The Scientific World Journal
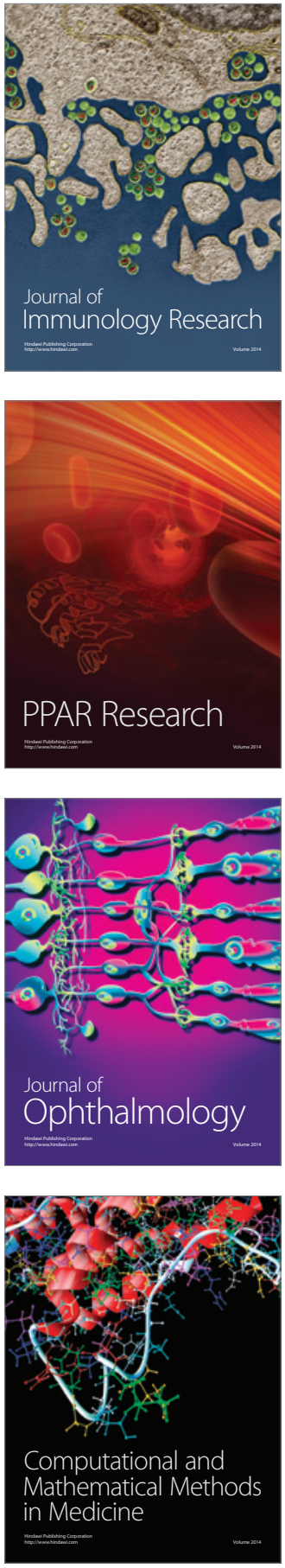

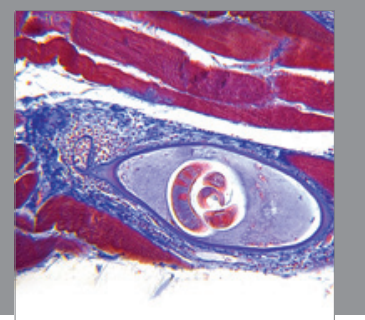

Gastroenterology

Research and Practice
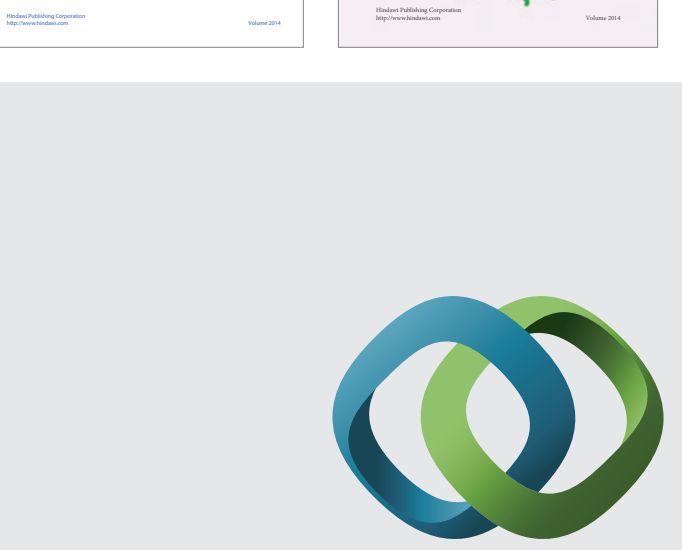

\section{Hindawi}

Submit your manuscripts at

http://www.hindawi.com
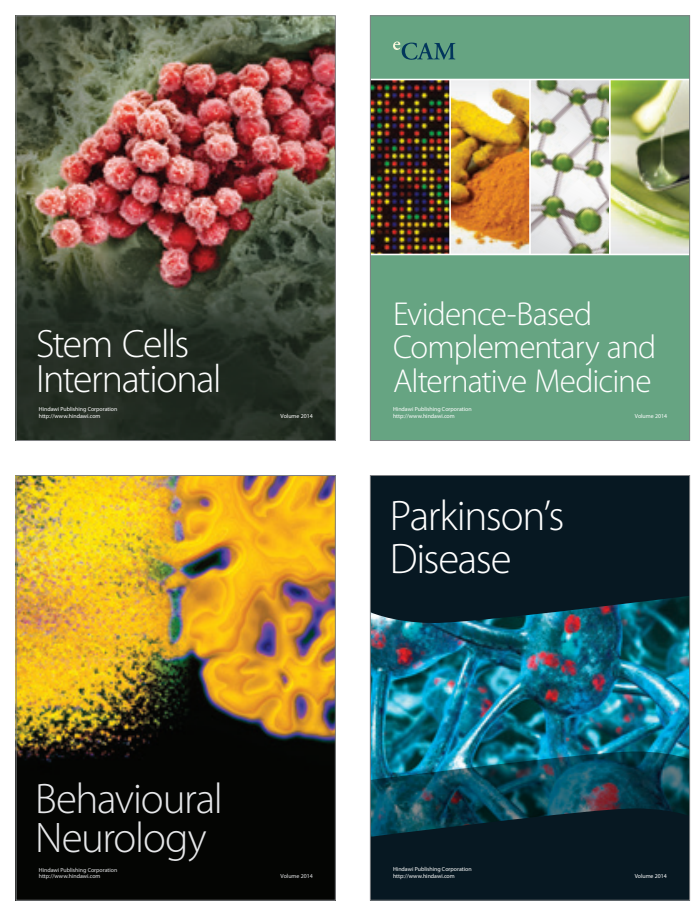

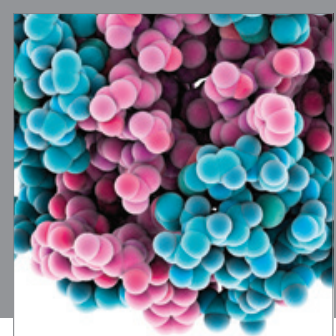

Journal of
Diabetes Research

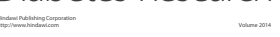

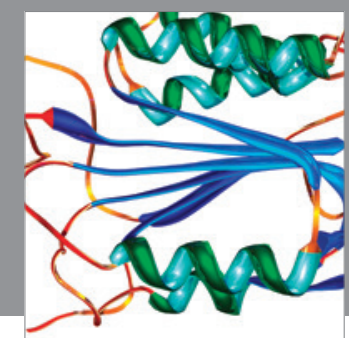

Disease Markers
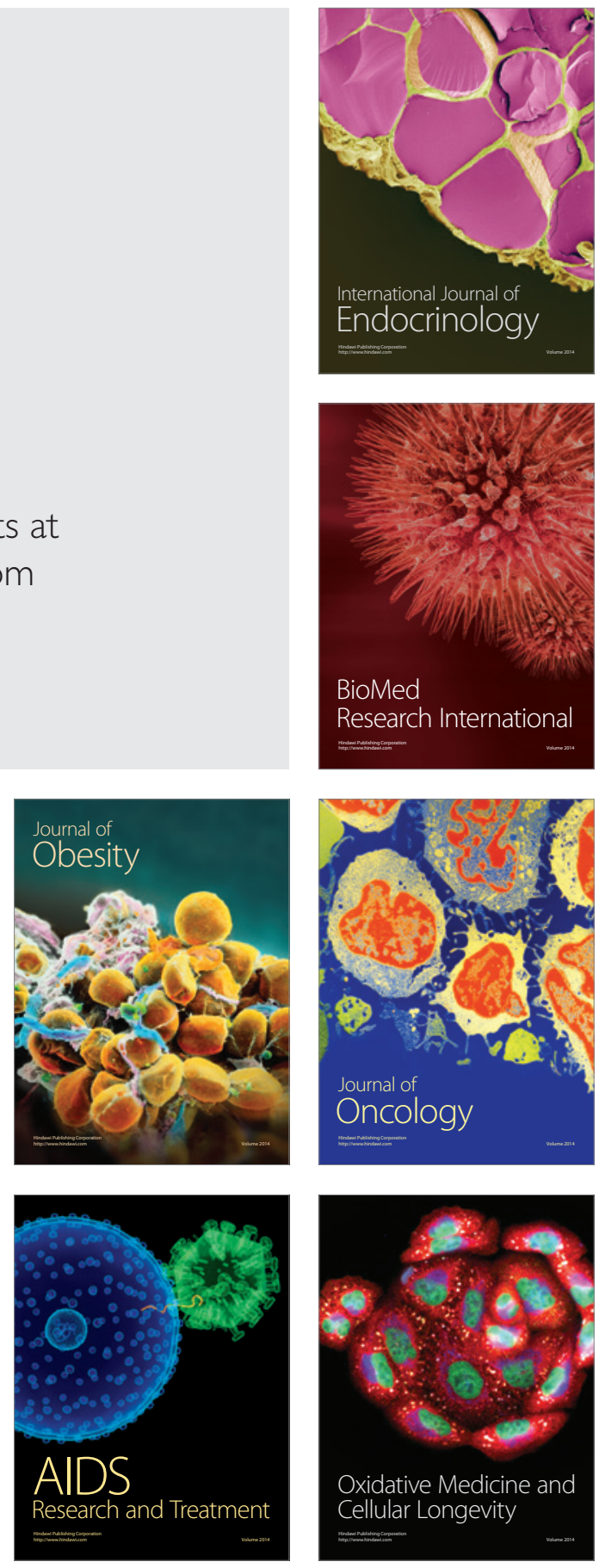